\title{
SEWING THREAD CONSUMPTION FOR CHAIN STITCHES OF CLASS 400 USING GEOMETRICAL AND MULTILINEAR REGRESSION MODELS
}

\author{
Sarah Malek ${ }^{1}$, Faouzi Khedher ${ }^{1}$, Dominique C. Adolphe ${ }^{2}$, Boubaker Jaouachi ${ }^{1}$ \\ 1 Laboratory of Textile Engineering, University of Monastir, Tunisia \\ 2 Laboratory of Physics and Textile Mechanics EA, University of Haute-Alsace, France \\ ${ }^{*}$ Corresponding author. E-mail: maleksarah88@gmail.com
}

\begin{abstract}
:
This work deals with determination of rapid and precise methods to predict the amount of sewing thread needed to sew a garment using different chain stitches of the class 400 (from 401 to 407 chain stitches). At first, to avoid unused stocks, sewing consumption value was determined using a geometrical method (based on different chain stitch shapes). The prediction of the sewing thread consumption was proposed as a function of the studied input parameters, which are fabric thickness, stitch density, yarn linear density, and stitch width. Then, a statistical method based on the multilinear regression was studied. Geometrical and statistical results were discussed. Based on the $R^{2}$ range, we concluded that the geometrical method is more accurate than the statistical one (from 98.16 to $99.19 \%$ and from 97.30 to $98.51 \%$, respectively). Thus, this result encourages industrialists to use geometrical models to predict thread consumption.
\end{abstract}

Also, all studied parameters, contributing to the sewing thread consumption behavior, were investigated and analyzed. The result shows that the most important parameters affecting thread consumption are stitch density followed by stitch width and fabric thickness. The yarn density has a low contribution on the thread consumption value.

\section{Keywords:}

Thread consumption, chain stitches of the class-400, geometrical models, multilinear regression models, input parameters

\section{List of abbreviations}

$a:$ Needle threads

$l:$ Looper thread

$e:$ Fabric thickness

$n:$ Stitch density expressed by stitch number/cm

$L$ : Sewing length

$d$ : Sewing thread diameter

$S L$ : Stitch length

Tex: Yarn linear density

$S_{w}:$ Sewing width

$X$ : Distance between two needles expressed in cm
$C_{401}$ : Thread consumption per centimeter of chain stitch type 401

$C_{402}$ : Thread consumption per centimeter of chain stitch type 402

$C_{406}$ : Thread consumption per centimeter of chain stitch type 406

$C_{T h}$ : Theoretical value of thread consumption

$C_{E x p}:$ Experimental value of thread consumption

$\hat{v}_{i}$ : Vector of the expected values of the response

$v_{i}$ : Vector of the observed values of the response

$\bar{v}:$ Average value of the answer 


\section{Introduction}

Considering that the sewing thread contributes significantly to the useful life of a product, it presents one of the most important components of a sewn product [1]. Sewing thread consumption is very interesting for the garment manufacturers and also for researchers. Thus, the sewing thread consumption topic helps manufacturers to estimate their consumed thread based on stitch type consumptions before launching their seaming process. For researchers, it is still difficult to evaluate the sewing thread amount required to stitch a garment and to predict it as a function of the most influential input parameters. Researchers are using different techniques whereas the industrialists are still looking for reliable, efficient, and fruitful solutions.

An important number of studies have investigated some garment problems to find a precise method allowing to objectively predicting the amount of sewing thread required to prepare garments [2-4]. However, the determination of the accurate required amount is still difficult. In fact, the consumptions of sewing thread depend on different input parameters, which include yarn count, mass, wastage, rate of breaks during sewing, etc., which makes it difficult to predict the required thread amount. Indeed, a little number of investigation have determined the relationship among the sewing machine parameters, the thread insertion inputs, the clothing morphology factors simultaneously, and the consumed thread [5,6]. Approximations and presumptions have been analyzed especially when stitch density, seam type, and material thickness are the main studied input parameters [7]. In fact, the thread consumption value cannot be considered, in any case, as standard for sewn garment such as jean pants, shirts, and jackets because the variable parameters change according to the presumed garment's style or type. In this context, Lauriol estimated waste percentage value of sewing thread $(10-15 \%)$, which should be added to the approximate values of consumed sewing thread [8].

Many researches are done, based on the different techniques, to predict the amount sewing thread consumption. For example, Rasheed considered the geometrical model to calculate the consumption of the sewing thread for 301 chain stitch [6]. Sharma et al. predicted the sewing thread consumption for chain stitch using regression models [9], and Vlinay et al. predicted the sewing thread consumption for lockstitch using regression models [10]. Also, Jaouachi et al. supplied an accurate method to predict the amount of sewing thread required to stitch a specific length of woven fabric using lockstitch (301) and chain stitch (401) using regression models, neural network, geometrical models, and fuzzy method [3, 11, 12]. Furthermore, Ghosh and Chavhan have determined a geometrical model of stitch length for lockstitch seam [13]. Jaouadi et al. compared thread consumption using both regression analysis and neural network [14]. Recently, Midha et al. have predicted sewing thread consumption for lockstitch using regression model [15]. Moreover, Rasheed et al. studied a geometrical model to calculate the consumption of sewing thread for 504 overedge stitch [1]. Gazzah et al. have developed a model, based on the 602 geometrical cover-stitches, to calculate the relative consumed sewing thread length [16].

According to literature, many previous works used the geometrical model, regression model, and neural network model to predict the amount of sewing thread consumption. However, the prediction of thread consumption has been studied for the $301,401,504,516$, and 602 stitches. To the best of our knowledge, there is no work in the literature that aimed to develop the geometrical relationships between the overall chain stitch types in the same class based on the geometrical models relative and stitch geometry consumptions (not two or three in the same class).

According to the studies already carried out, among overall stitch types of class 400 , only the thread consumption of chain stitch type 401 has been studied. For this reason, we have investigated the most used chain stitch types in class 400 to sew garments in this study. Undoubtedly, different clothes are seamed based mostly on these studied stitch types, such as denim garments, hems of knitwear, for the laying of mesh fabric and lace underwear and lingerie.

In this work, a determination of the sewing thread required amount to stitch a specific length of woven fabric using seven chain stitch types of class 400 (from 401 to 407 ) has been conducted. To help industrialists estimate accurately the consumed amount of sewing thread, two methods are proposed and compared. Therefore, the geometrical method and the multilinear regression method are investigated. In the last part, the effect of each input parameter has been studied and discussed and to clarify the most significant factors to regulate before launching production to industrialists.

\section{Material and methods}

\subsection{Fabric properties}

In our study, six commercial denim fabrics were chosen. These fabrics have different characteristics, namely thicknesses and blend compositions. The denim fabrics were prepared on a SULZER P7300 weaving loom projectile with 3/1 twill structure. As denim fabrics are generally made of cotton, cotton/elastane, or cotton/polyester/elastane, these specific compositions are considered.

Table 1 presents these samples and their properties. For each sample, each test is repeated five times according to the French standards.

\subsection{Sewing thread properties}

In this study, two commercial sewing threads, commonly used for sewing denim fabrics, were chosen. The thread selection was based on their linear densities, which can affect the used amount of sewing thread. The sewing thread linear densities were chosen according to NFG 07-117 [17]. The counts of the threads were 63.5 and 95 tex. 
Table 1. Fabric properties

\begin{tabular}{|c|c|c|c|c|c|c|c|c|c|c|c|}
\hline \multirow{2}{*}{$\begin{array}{c}\text { Parameter } \\
\begin{array}{c}\text { Fabric } \\
\text { composition n }\end{array}\end{array}$} & \multirow{2}{*}{$\begin{array}{c}\text { Warp yarn } \\
\text { density } \\
\text { (ends/cm) }\end{array}$} & \multirow{2}{*}{$\begin{array}{c}\text { Weft yarn } \\
\text { density } \\
\text { (picks/cm) }\end{array}$} & \multicolumn{2}{|c|}{$\begin{array}{c}\text { Linear } \\
\text { density (tex) }\end{array}$} & \multicolumn{2}{|c|}{$\begin{array}{c}\text { Mass per } \\
\text { square } \\
\text { meter }\end{array}$} & \multicolumn{2}{|c|}{ Thickness } & \multicolumn{3}{|c|}{ Tensile properties } \\
\hline & & & Warp & Weft & $\begin{array}{l}\text { Mass } \\
\left(\mathrm{g} / \mathrm{m}^{2}\right)\end{array}$ & $\begin{array}{l}\text { CV } \\
(\%)\end{array}$ & $\begin{array}{l}\text { Thickness } \\
\text { (mm) }\end{array}$ & $\begin{array}{l}\text { CV } \\
(\%)\end{array}$ & $\begin{array}{l}\text { Breaking } \\
\text { force }(N)\end{array}$ & $\begin{array}{c}\text { Elongation at } \\
\text { break (\%) }\end{array}$ & $\begin{array}{l}\text { CV } \\
(\%)\end{array}$ \\
\hline \multirow{2}{*}{1} & 26 & 17 & 11.9 & 20 & 323 & 1.9 & 1.2 & 1.4 & 413.63 & 12.66 & 1.4 \\
\hline & 30 & 22 & 12.5 & 16.67 & 387 & 2.1 & 1.04 & 1 & 1071.90 & 17.89 & 2.1 \\
\hline \multirow{2}{*}{2} & 32 & 17 & 11.36 & 14.92 & 445 & 1.5 & 0.96 & 1.4 & 894.26 & 17.40 & 0.8 \\
\hline & 29 & 22 & 12.5 & 27.78 & 342 & 1.2 & 0.90 & 1.6 & 624.21 & 30.45 & 1.2 \\
\hline \multirow{2}{*}{3} & 31 & 21 & 12.5 & 27.78 & 334 & 1.6 & 0.85 & 1.2 & 557.24 & 33.64 & 0.6 \\
\hline & 28 & 22 & 14.29 & 27.78 & 328 & 0.9 & 0.75 & 2.1 & 528.13 & 25.12 & 2.4 \\
\hline
\end{tabular}

Fabric composition 1: $100 \%$ cotton warp and weft threads.

Fabric composition 2: 100\% cotton warp yarn and weft yarn in 95\% cotton and $5 \%$ elastane.

Fabric composition 3: $100 \%$ cotton warp yarn and weft yarn in $71 \%$ cotton, $5 \%$ elastane, and $24 \%$ polyester.

Table 2 presents the sewing threads' properties.

Table 2. Sewing threads properties

\begin{tabular}{|c|c|c|}
\hline Properties & Thread $\mathbf{N}^{\circ} \mathbf{1}$ & Thread N $^{\circ} \mathbf{2}$ \\
\hline Linear density (tex) & 63.50 & 95.00 \\
\hline Number of strands & 3 & 3 \\
\hline Composition & $100 \%$ PES & $100 \%$ PES \\
\hline Twist direction & S & S \\
\hline Breaking force $(N)$ & 24.23 & 26.43 \\
\hline Breaking elongation $(\%)$ & 21.12 & 16.90 \\
\hline Rigidity (N/m) & 389.12 & 536.59 \\
\hline Twist/m & 353.00 & 294.00 \\
\hline Tenacity (cN/tex) & 38.16 & 27.82 \\
\hline
\end{tabular}

PES, polyester.

\subsection{Methods used for the thread consumption prediction}

In this study, the geometrical and the multilinear regression methods are considered. We tested the most useful chain stitch of class 400 in sewn product industry. Chain stitch class 400 is the class used in most types of sewn products. Its thread consumption is as high as class 500 . Both stitch classes are usually used for sewing denim garments (such as trousers, shirts, jacket, knitwear, mesh fabric, lingerie, etc.). For this reason, seven types of stitch are chosen. In this work, we try to establish developed models to determine consumed thread based on the chain stitch of class 400 . Thus, making one of the originalities of the present study, because until now there is no work dealing with overall types of stitch in the same class.

In fact, seven types of stitch for the class 400 were considered. These stitches are as follows:
(3) Chain stitch 402, two needles, three threads

(6) Chain stitch 403 , three needles, four threads

(9) Chain stitch 404, two threads zig-zag

Chain stitch 405, two needles, three threads zig-zag

(15) Chain stitch, two needles, three threads with simple recovery 406

(18) Chain stitch, three needles, four threads with simple recovery 407

Nevertheless, for the multilinear regression, using MINITAB-17 software, a statistical method based on the experimental design was applied to conduct this modeling technique objectively. Moreover, individual effects of input parameter were determined and analyzed. For both geometric and statistical methods, five input parameters with their levels were taken into account (fabric thickness [six levels], stitch density [three levels], yarn count [two levels], stitch width [three levels], and gap between two needles [two levels]). The predicted thread consumption is extremely related to the stitched fabric plies or layers. Considering that it is highly complicated to optimize all parameter effects during the sewing process, in this work, all samples are sewn using two fabrics plies.

\section{Results and discussion}

\subsection{Geometrical model}

In this part, the stitch modeling was realized using mathematical calculation based on geometrical shapes of chain stitch geometries and some assumptions to facilitate the consumed thread calculation. Two software are used to represent the overall shape geometries of chain stitch class 400 , which are 3ds Max 2014 and keyshot 5. 
In our case, we must indicate that:

- The cross-section of threads is circular and incompressible in nature.

- In all cases, the needle threads and looper threads are the same.

\subsubsection{Case of chain stitch type 401}

Figure 1a presents the actual seam configuration and the positions of interlacing points formed by needle and looper threads. Figure $1 \mathrm{~b}$ indicates a stitch configuration of a chain stitch seam with a repeating unit. Thus, the thread length of a unit cell is supposed the same throughout the seam length for all repeated units. From the geometrical representation of each studied chain stitch, the length of the sewing thread (from needle threads $[a]$ and looper thread $[l]$ ) was calculated for each stitch based on simple mathematical principles (sum, shape calculation using the basic mathematical relationships) while considering the circular section of thread, incompressible threads, and incompressible fabrics. In fact, it was assumed that the yarn is circular and incompressible in nature. Also, the fabric is incompressible in nature. The model is two dimensional in nature.

The amount of sewing thread $C_{401}$ needed for the 401 chain stitch, whose geometry is represented in Figure $1 \mathrm{~b}$, is estimated by the following Eq. (1).

$C_{401}=\left(C_{401 a}+C_{4011}\right) \times n \times L$

where $C_{401 a}$ is the consumption of needle thread and $C_{401 l}$ is the consumption of looper thread.

Where

$C_{401 a}=\left(\frac{1}{n}-d\right)+4 e+\frac{1}{2}\left(\pi \times\left(d+\frac{d}{2}\right)\right)+2 \times \frac{d}{2}$

With $S L=\frac{1}{n}-d$

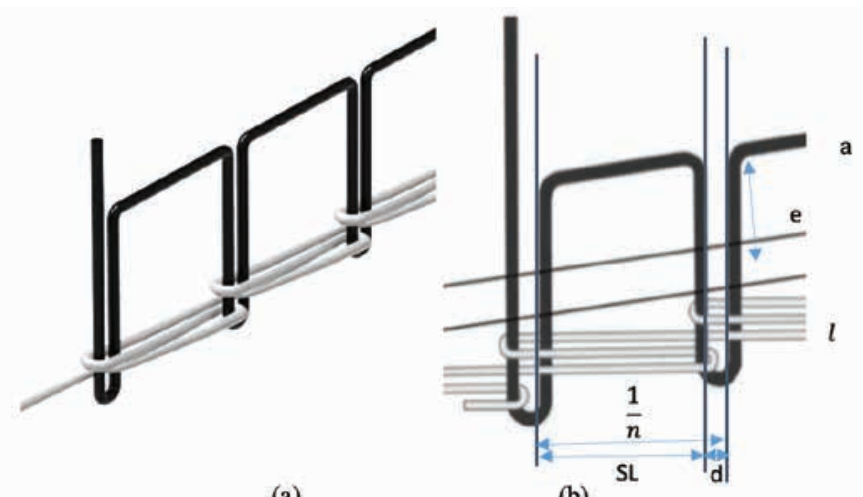

(a)

(b)

Figure 1. Geometrical model of chain stitch type 401 presented in two dimensions (a) and three dimensions (b).

$$
\begin{aligned}
& \text { Then } C_{401 a}=S L+4 e+\frac{1}{-}\left(\pi \times\left(\frac{3 \times d}{-}\right)\right)+d \\
& C_{401 l}=3 \times S L+2 \times \frac{1}{2}\left(\pi \times\left(\frac{3 \times d}{2}\right)\right)+3 \times d
\end{aligned}
$$

To calculate a sewing thread diameter supposed as circular $(d)$, we used the general equation proposed by Sohanur [18] as a function of the thread linear density Tex (see Equation 6).

$d(\mathrm{~cm})=\frac{0.375}{100} \sqrt{\mathrm{Tex}}$

\subsubsection{Case of chain stitch type 402}

The amount of sewing thread $C_{402}$ needed for the 402 chain stitch, whose geometry is represented previously in Figure 2 , is estimated by the following Eq. (7):

$$
C_{402}=\left(C_{402 a}+C_{402 l}\right) \times n \times L
$$

where $C_{402 a}$ is the consumption of needle thread and $C_{402 l}$ is the consumption of looper thread.

Regarding that chain stitch type 402 double that of chain stitch type 401, then

$$
C_{402 a}=\left(S L+4 e+\frac{1}{2}\left(\pi \times\left(d+\frac{d}{2}\right)\right)+2 \times \frac{d}{2}\right) \times 2
$$

$$
=\left(S L+4 e+\frac{1}{2}\left(\pi \times \frac{3 \times d}{2}\right)+d\right) \times 2
$$
As well as $Z$ distance is equal to $\left(\left(\frac{1}{n}\right)^{2}+S_{w}{ }^{2}\right)^{1 / 2}$, and $S_{W}$
is repeated three times.

Then,

$$
\begin{aligned}
& C_{402 l}=S L+\frac{1}{n}+\left(\left(\frac{1}{n}\right)^{2}+S_{w}^{2}\right)^{1 / 2}+3 \times S_{w}+\frac{d}{2} \times 6 \\
& =S L+\frac{1}{n}+\left(\left(\frac{1}{n}\right)^{2}+S_{w}{ }^{2}\right)^{1 / 2}+3 \times S_{w}+3 \times d
\end{aligned}
$$

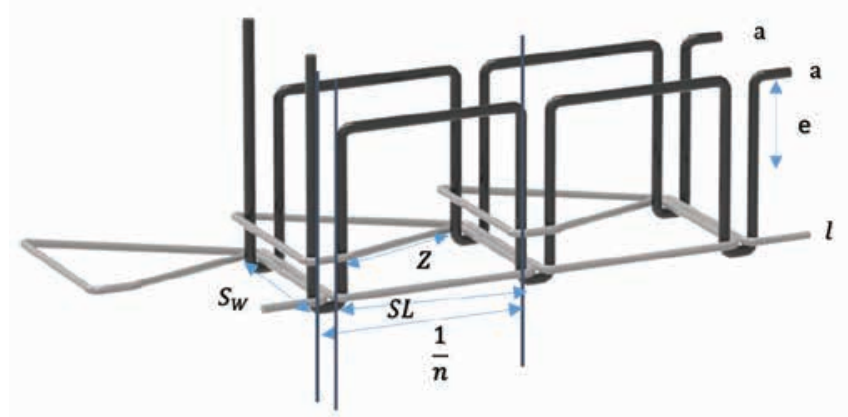

Figure 2. Geometrical model of chain stitch type 402. 


\subsubsection{Case of chain stitch type 403}

The amount of sewing thread $C_{403}$ needed for the 403 chain stitch, whose geometry is represented previously in Figure 3 , is estimated by the following Eq. (12):

$C_{403}=\left(C_{403 a}+C_{403 l}\right) \times n \times L$

where $C_{403 a}$ is the consumption of needle thread and $C_{403 l}$ is the consumption of looper thread.

Where

$$
\begin{aligned}
& C_{403 a}=\left(S L+4 e+\frac{1}{2}\left(\pi \times\left(d+\frac{d}{2}\right)\right)+2 \times \frac{d}{2}\right) \times 3 \\
& =\left(S L+4 e+\frac{1}{2}\left(\pi \times \frac{3 \times d}{2}\right)+d\right) \times 3 \\
& C_{403 l}=S L+\frac{1}{n}+\left(\left(\frac{1}{n}\right)^{2}+\left(2 \times S_{w}\right)^{2}\right)^{1 / 2}+6 \times S_{w}+\frac{d}{2} \times 6
\end{aligned}
$$$$
=S L+\frac{1}{n}+\left(\left(\frac{1}{n}\right)^{2}+\left(2 \times S_{w}\right)^{2}\right)^{1 / 2}+6 \times S_{w}+3 \times d
$$

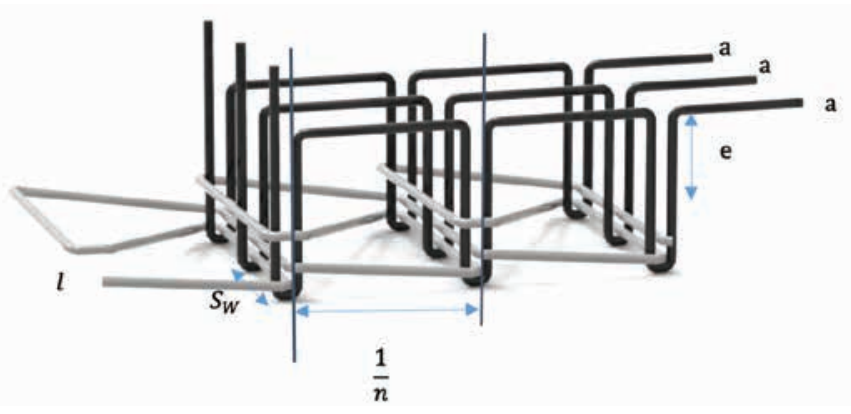

Figure 3. Geometrical model of chain stitch type 403.

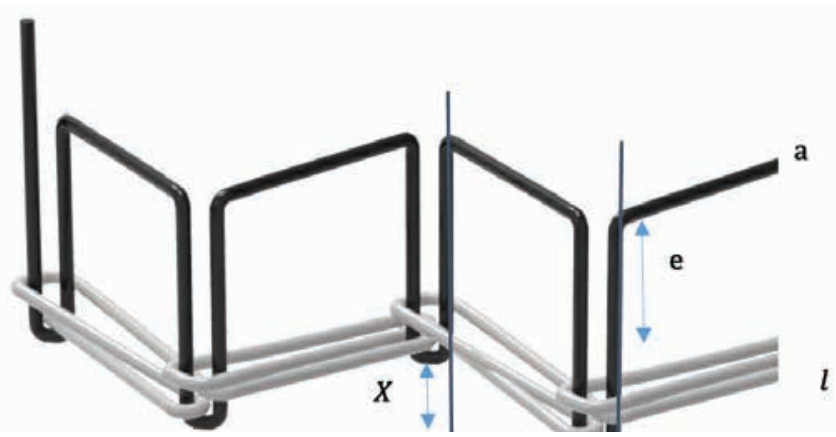

Figure 4. Geometrical model of chain stitch type 404.

\subsubsection{Case of chain stitch type 404}

The amount of sewing thread $C_{404}$ needed for the 404 chain stitch, whose geometry is represented previously in Figure 4 , is estimated by the following Eq. (17):

$C_{404}=\left(C_{404 a}+C_{404 l}\right) \times n \times L$

where $C_{404 a}$ is the consumption of needle thread and $C_{404 l}$ is the consumption of looper thread.

Where

$C_{404 l}=\left((S L)^{2}+X^{2}\right)^{1 / 2}+4 \times e+\frac{1}{2}\left(\pi \times\left(d+\frac{d}{2}\right)\right)+2 \times \frac{d}{2}$

$=\left((S L)^{2}+X^{2}\right)^{1 / 2}+4 \times e+\frac{1}{2}\left(\pi \times \frac{3 \times d}{2}\right)+d$

$C_{404 l}=3 \times\left(\left(\frac{1}{n}\right)^{2}+X^{2}\right)^{1 / 2}+3 \times d+2 \times \frac{1}{2}\left(\pi \times\left(d+\frac{d}{2}\right)\right)$

$=3 \times\left(\left(\frac{1}{n}\right)^{2}+X^{2}\right)^{1 / 2}+3 \times d+\left(\pi \times \frac{3 \times d}{2}\right)$

\subsubsection{Case of chain stitch type 405}

The amount of sewing thread $C_{405}$ needed for the 405 chain stitch, whose geometry is represented previously in Figure 5 , is estimated by the following Eq. (22):

$$
C_{405}=\left(C_{405 a}+C_{405 l}\right) \times n \times L
$$

where $C_{405 a}$ is the consumption of needle thread and $C_{405 l}$ is the consumption of looper thread.

$$
\begin{aligned}
& C_{405 a}=\left(\left((S L)^{2}+X^{2}\right)^{1 / 2}+4 \times e+\frac{1}{2}\left(\pi \times\left(d+\frac{d}{2}\right)\right)+2 \times \frac{d}{2}\right) \times 2 \\
& =\left(\left((S L)^{2}+X^{2}\right)^{1 / 2}+4 \times e+\frac{1}{2}\left(\pi \times \frac{3 \times d}{2}\right)+d\right) \times 2
\end{aligned}
$$

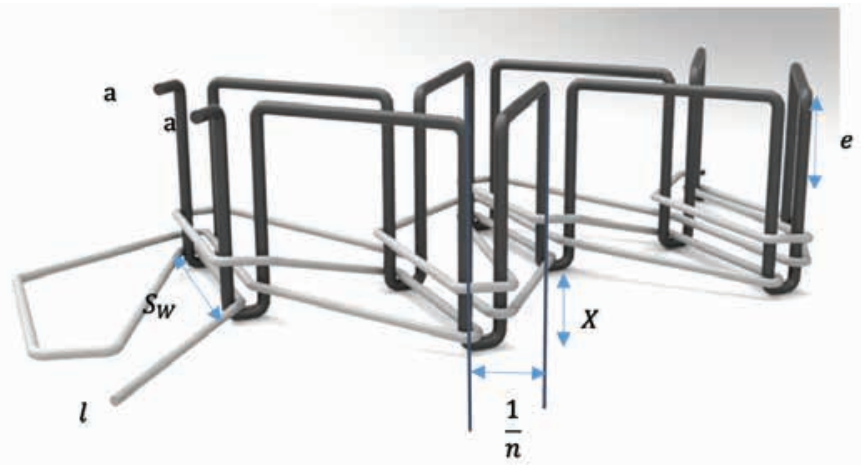

Figure 5. Geometrical model of chain stitch type 405. 


$$
\begin{aligned}
& C_{40 s 1}=\left((S L)^{2}+X^{2}\right)^{\prime / 2}+\left(\left(\frac{1}{n}\right)^{2}+X^{2}\right)^{1 / 2}+\left(\left((S L)^{2}+X^{2}\right)^{1 / 2}+S_{w}^{2}\right)^{2 / 2}+3 \times S_{w}+\frac{d}{2} \times 6 \\
& =\left((S L)^{2}+X^{2}\right)^{\prime / 2}+\left(\left(\frac{1}{n}\right)^{2}+X^{2}\right)^{1 / 2}+\left(\left((S L)^{2}+X^{2}\right)^{1 / 2}+S_{w}{ }^{2}\right)^{\prime / 2}+3 \times S_{w}+3 \times d
\end{aligned}
$$

\subsubsection{Case of chain stitch type 406}

The amount of sewing thread $C_{406}$ needed for the 406 chain stitch, whose geometry is represented previously in Figure 6 , is estimated by the following Eq. (27):

$$
C_{406}=\left(C_{406 a}+C_{406 l}\right) \times n \times L
$$

where $C_{406 a}$ is the consumption of needle thread and $C_{406 l}$ is the consumption of looper thread.

$$
\begin{aligned}
& C_{406 a}=\left(S L+4 e+\frac{1}{2}\left(\pi \times\left(d+\frac{d}{2}\right)\right)+2 \times \frac{d}{2}\right) \times 2 \\
& =\left(S L+4 e+\frac{1}{2}\left(\pi \times \frac{3 \times d}{2}\right)+d\right) \times 2 \\
& C_{406 t}=3 \times \frac{1}{n}+S L+\left(\left(\frac{1}{n}\right)^{2}+S_{w}^{2}\right)^{1 / 2}+S_{w}+2 \times \frac{1}{2}\left(\pi \times\left(d+\frac{d}{2}\right)\right)+3 \times \frac{d}{2} \\
& =\frac{3}{n}+S L+\left(\left(\frac{1}{n}\right)^{2}+S_{w}^{2}\right)^{1 / 2}+S_{w}+\left(\pi \times \frac{3 \times d}{2}\right)+\frac{3 \times d}{2}
\end{aligned}
$$

\subsubsection{Case of chain stitch type 407}

The amount of sewing thread $C_{407}$ needed for the 407 chain stitch, whose geometry is represented previously in Figure 7 , is estimated by the following Eq. (32):

$$
C_{407}=\left(C_{407 a}+C_{407 l}\right) \times n \times L
$$

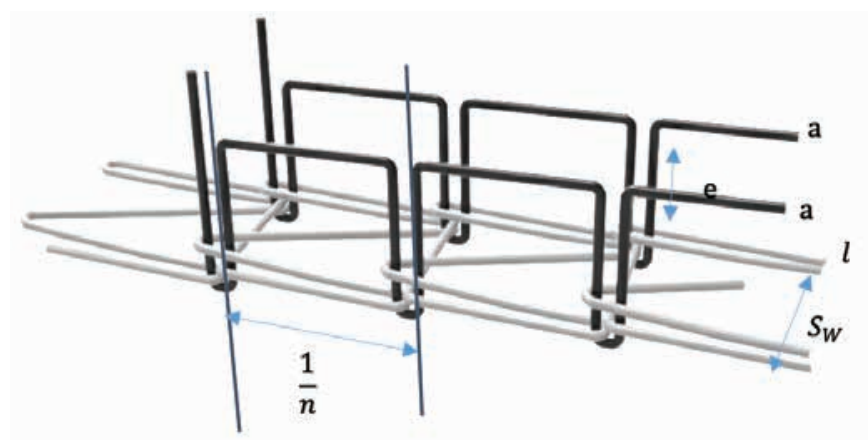

Figure 6. Geometrical model of chain stitch type 406. where $C_{407 a}$ is the consumption of needle thread and $C_{407 l}$ is the consumption of looper thread.

Where

$$
\begin{aligned}
& C_{407 a}=\left(S L+4 e+\frac{1}{2}\left(\pi \times\left(d+\frac{d}{2}\right)\right)+2 \times \frac{d}{2}\right) \times 3 \\
& =\left(S L+4 e+\frac{1}{2}\left(\pi \times \frac{3 \times d}{2}\right)+d\right) \times 3
\end{aligned}
$$

$C_{407 t}=4 \times \frac{1}{n}+S L+2 \times\left(\left(\frac{1}{n}\right)^{2}+S_{w}{ }^{2}\right)^{1 / 2}+2 \times S_{w}+2 \times \frac{1}{2}\left(\pi \times\left(d+\frac{d}{2}\right)\right)+6 \times \frac{d}{2}$

$$
=\frac{4}{n}+S L+2 \times\left(\left(\frac{1}{n}\right)^{2}+S_{w}{ }^{2}\right)^{1 / 2}+2 \times S_{w}+\left(\pi \times \frac{3 \times d}{2}\right)+3 \times d
$$

So as to verify these models, the most useful stitches for clothing manufacture are chosen. Thus 401,402 , and 406 chain stitches are considered to verify practically the determined geometrical models. This choice is based also on the availability of sewing machines. In fact, if the result is verified for a definite number of stitches, it will be verified for the rest of models. For both chain stitch types 401 and 402, the used sewing machines type is JUKI MH-380 double chain stitch (speed: $2000 \mathrm{rp} / \mathrm{min}$, two needles). However, for the simple recovery chain stitch type 406 with two needles and four threads, Siruba industrial sewing machine model F007 J (speed $=2000 \mathrm{rp} / \mathrm{min}$ ) was used.

By varying the different factors, 36 combinations will be considered for 401 chain stitch (combination of three input parameters with $e$ (six levels), $n$ (two levels), and tex (two levels) so the tests number will be equal to $6 \times 2 \times 2=36$ tests) and 72 combinations for 402 and 406 chain stitches (combination of four input parameters with $e$ (six levels), $n$ (two levels), tex (two levels), and $S_{w}$ (two levels) so the number of tests will be equal to $6{ }^{\prime} 2^{\prime} 2^{\prime} 2=72$ tests). Each test (combination) is repeated five times. Thus, 180 samples were prepared by varying the different factors for (401) chain stitches, 360 samples were prepared for 402 and 406 chain stitches, respectively.

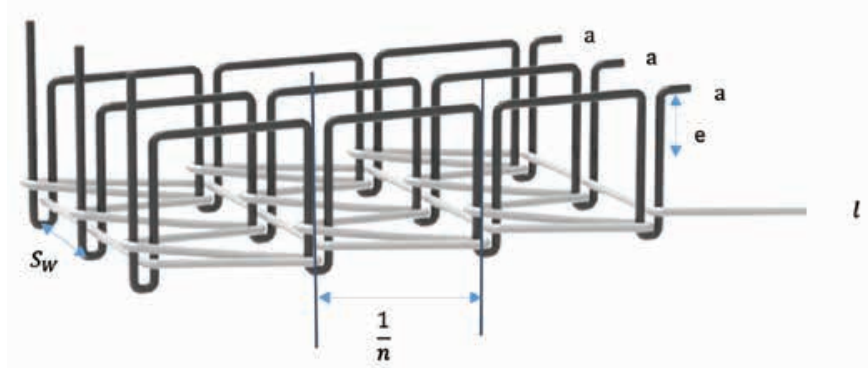

Figure 7. Geometrical model of chain stitch type 407. 
Table 3 presents the levels of each factor for preparing the samples of each type of chain stitch of class 400 .

Table 3. Levels of each factor

\begin{tabular}{|c|c|c|c|c|}
\hline $\begin{array}{c}\text { Input } \\
\text { parameter }\end{array}$ & $\boldsymbol{e}$ (cm) & $\begin{array}{c}\boldsymbol{n} \\
\text { (stitches/ } \\
\mathbf{c m})\end{array}$ & $\begin{array}{c}\boldsymbol{T e x} \\
\text { (tex) }\end{array}$ & $\begin{array}{c}S_{\boldsymbol{w}} \\
\text { (cm) }\end{array}$ \\
\hline Level 1 & 0.120 & 3 & 63.5 & 0.48 \\
\hline Level 2 & 0.104 & 4 & 95 & 0.65 \\
\hline Level 3 & 0.096 & 5 & - & - \\
\hline Level 4 & 0.090 & - & - & - \\
\hline Level 5 & 0.085 & - & - & - \\
\hline Level 6 & 0.075 & - & - & - \\
\hline
\end{tabular}

No value of parameter, which should be mentioned.

Practically, the samples were prepared, and a $10 \mathrm{~cm}$ seam length of each sample was considered. Then, the seam was unstitched to get the needle and looper thread consumed in $5 \mathrm{~cm}$ length. After unstitching, the sewed thread length was determined to measure the value of the consumed thread per $\mathrm{cm}$.

The average absolute relative error, $\overline{\text { Error }}$ [Eq. (37)] between the theoretical $\left(C_{T h}\right)$ and experimental values $\left(C_{E x p}\right)$ was determined to improve the obtained results.

$$
\overline{\text { Error }}=\frac{\left|C_{T h}-C_{E x p}\right|}{C_{E x p}} * 100
$$

According to Gazzeh, the limit of the mean absolute error values used in the modeling is estimated to be $6 \%$ [19].

Table 4 presents the average error for each type of chain stitch of class 400 .

Table 4. Average error

\begin{tabular}{|c|c|c|c|}
\hline Stitch & 401 & 402 & 406 \\
\hline Error (\%) & 1.46 & 1.42 & 2.78 \\
\hline
\end{tabular}

Based on our finding, the average absolute error does not exceed $2.80 \%$. Regarding the findings, the error values are much lower than $6 \%$. Thus, the result is widely verified, and the studied models are well justified and the findings are highly significant.

The comparison between the experimental and theoretical values showed a good agreement between geometrical and experimental consumption values. In fact, the coefficient of regression ranged from 98.16 to $99.19 \%$ (Figure 8), which reflects significant and efficient relationships between experimental and theoretical findings.

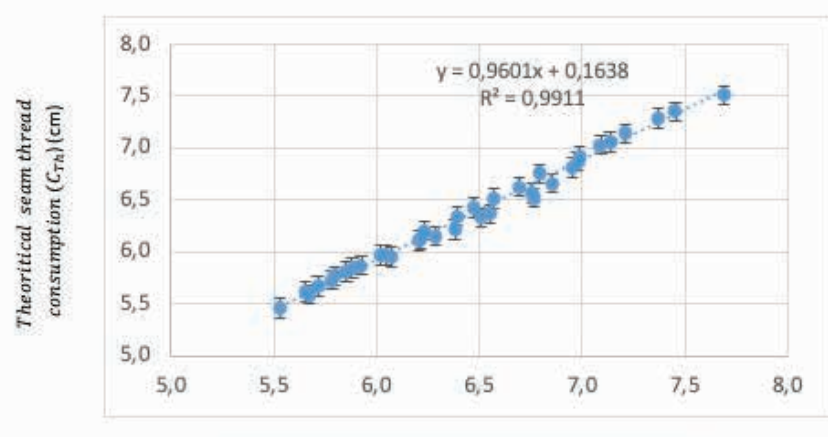

Experimental seam thread consumption $\left(C_{E x p}\right)(\mathrm{cm})$

(a)

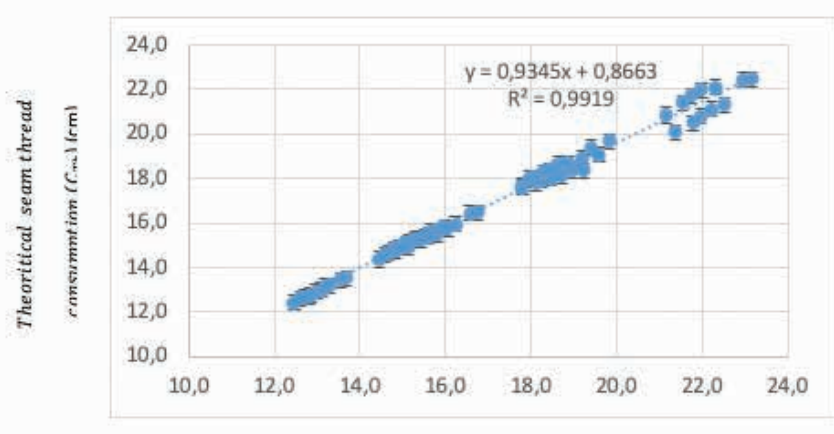

Experimental seam thread consumption $\left(C_{E x p}\right)(\mathrm{cm})$

(b)

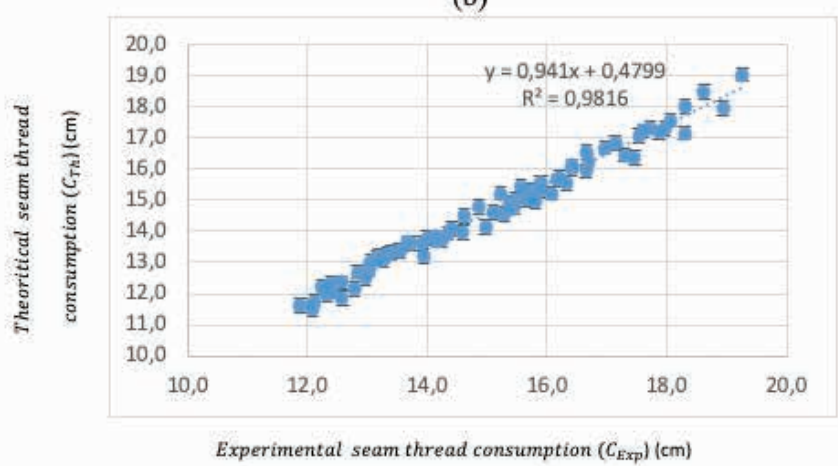

(c)

Figure 8. Theoretical consumption evolution as a function of experimental results for chain stitch type 401 (a), 402 (b), and 406 (c).

Figure $8 a-c$ has shown the comparisons between the theoretical and experimental consumption values for chain stitch type 401, 402, and 406. In fact, the experimental thread consumption ( $x$-axis) is plotted against the theoretical thread consumption ( $y$-axis). Linear regression is applied, and a line is fitted to the data. The $R^{2}$ value of the fitted line is equal to $0.9911,0.9919$, and 0.9816 , which means that the difference between the two data sets is very small. The high $R^{2}$ value verifies the model accuracy and depicts also that the model has taken into account all the variables on which the thread consumption depends. This result is in accordance with Rasheed et al. who concluded, using geometrical models, that the difference between the actual and predicted thread consumptions, for 504 over edge stitch, sets is very small $\left(R^{2}\right.$ value is equal to 0.992 ) [1]. 
So, regarding that the error rate is very low and the regression coefficient values are very high (from 98.16 to $99.21 \%$ ), it was concluded that the obtained geometrical models are well fruitful and could be recommended for the prediction of sewing thread consumption in the experimental design of interest.

Considering that our results are well verified and validated for the consumption of the stitch 401, 402, and 406, we can validate the other chain stitches in this class, which are 403 , 404, 405, and 407.

\subsection{Multilinear regression models}

The multilinear regression method was studied to compare its results with those obtained using the geometrical method, in order to help industrialists to use the best method to predict the consumed thread to sew denim garments.

Based on the experimental design, a statistical method was applied to faithfully estimate the suitable amount of thread based on the different chain stitch types and study the effect of each investigated input parameter. The multilinear regression models were determined using MINITAB-17 software. The input parameter effects are analyzed. Tables 3 and 4 present the studied input parameters as well as their levels. The different models for chain stitch type 401,402 , and 406 are considered as shown in Table 5.

Table 5. Multilinear regression models for different chain stitches of class 400

\begin{tabular}{|c|c|}
\hline Equation & $\begin{array}{c}\boldsymbol{R}^{2} \\
(\%)\end{array}$ \\
\hline$C_{401}=2,024+14,681 e+0,5881 n+0,007496 \mathrm{Tex}$ & 97.66 \\
\hline$C_{402}=-6.479+29.21 e+2.9890 n+0.00443 T e x+14.744 S_{w}$ & 98.51 \\
\hline$C_{406}=-1.586+31.60 e+2.0110 n+0.01091 T e x+7.725 S_{w}$ & 97.30 \\
\hline
\end{tabular}

The regression coefficient value $\left(R^{2}\right)$ reflects the significance and relevance of the developed models. This regression coefficient is ranged from 0 to $100 \%$. Thus, the closer it is to $100 \%$, the more significant the model is and vice versa [3].

Furthermore, it is important to analyze the statistical analysis variance. Hence, it is possible to evaluate the importance of different parameters based on the $p$-value statistical coefficient. Indeed, three conditions are presented:

- $\quad$ if $p$-value is null, then the parameter is very significant.

- if $p$-value is ranged from 0 to 0.05 , then the parameter is significant.

- if $p$-value is higher than 0.05 , then the parameter is negligible.

The $p$-values for each multilinear regression model are presented in Table 6.

Table 6. $p$-value for each studied model

\begin{tabular}{|c|c|c|c|c|}
\hline Parameter & $\boldsymbol{e}$ & $\boldsymbol{n}$ & $\boldsymbol{T e x}$ & $\boldsymbol{S}_{\boldsymbol{W}}$ \\
\hline 401 model & 0.000 & 0.000 & 0.000 & - \\
\hline 402 model & 0.000 & 0.000 & 0.075 & 0.000 \\
\hline 406 model & 0.000 & 0.000 & 0.000 & 0.000 \\
\hline
\end{tabular}

No value of parameter that should be mentioned.

According to the conditions mentioned above, each parameter having a $p$-value higher than 0.05 is neglected. Then, as shown in Table 6, all parameters are not neglected unless the linear density for 402 multilinear equation. Therefore, the multilinear regression models presented in Table 7 are considered.

Table 7. Multilinear regression models for 401,402 , and 406 chain stitches

\begin{tabular}{|c|c|}
\hline Equation & $\begin{array}{c}\boldsymbol{R}^{2} \\
(\%)\end{array}$ \\
\hline$C_{401}=2,024+14,681 e+0,5881 n+0,007496 T e x$ & 97.66 \\
\hline$C_{402}=-6.128+29.21 e+2.9890 n+14.744 S_{w}$ & 98.48 \\
\hline$C_{406}=-1.586+31.60 e+2.0110 n+0.01091 T e x+7.725 S_{w}$ & 97.30 \\
\hline
\end{tabular}

Considering the range of $\mathrm{R}^{2}$ values obtained (from 97.30 to $98.51 \%$ ), it may be considered as reliable and significant. Therefore, this $\mathrm{R}^{2}$ range shows the effectiveness of the statistical method to predict the suitable consumption value. Moreover, to determine consumed thread, industrialists can use statistical results for the estimation of the consumed amount using multilinear regression models.

Compared with the geometrical modeling technique, the statistical method remained less accurate, powerful, and predictable regarding its $R^{2}$ range (from 98.16 to $99.21 \%$ and from 97.30 to $98.51 \%$ for geometrical and statistical models, respectively). Regarding the mathematical modeling, the geometrical method takes into account some hypotheses, such as considering sewing thread section circular, fabric incompressible, the width of stitch constant, and threads incompressible, which makes the consumed thread calculation more precise and independent of parameters considered in the statistical technique variables. Comparing the two studied methods, we found that the geometrical one is found more effective than the regressive method and fitted the experimental results widely. This result can be explained by the hypothesis used to simplify the determination of an approximate consumption value. The geometrical models allow a fruitful prediction of the consumed sewing thread amount for the experimental design of interest. In fact, the calculation of the sewing thread consumption based on different input parameters generates some errors during stitching and unstitching thread and explains the range of the regression coefficient from 98.16 to $99.21 \%$. Thus, all measures can be subject to error taking into account wastage, reproducibility of unstitching steps, and so on. Therefore, the geometric modeling technique gives more 
accurate results than the statistical method. These findings seem in accordance with those in the literature $[1,6]$.

\subsection{Effects of input parameters}

In this part, we determined the effect of each input parameter. The influence of fabric thickness, stitch density, yarn linear density, and the stitch width for the different studied stitches (401, 402, and 406) are presented in Figures 11-13.

\subsubsection{Fabric thickness $(e)$ effect}

Referring to Figure $9 a-c$, it is notable that the increase of fabric thickness from 0.075 to $0.123 \mathrm{~mm}$, increases thread consumption by $10.91,8.13$, and $10.43 \%$, for 401,402 , and 406 chain stitches, respectively. Thus, the fabric thickness does not have a very important effect, but it remains an influential factor influencing thread consumption. In fact, the increase of fabric thickness increases thread consumption for the three studied chain stitches. In addition, the increase of fabric thickness increases seam thickness as well as thread consumption. Therefore, less thick fabric requires less thread, but thicker fabric requires more thread. This result is in accordance with Rasheed et al. and Jaouachi et al.'s finding that consumption values increase with the increase of fabric thickness. This means that the thickness of fabric samples affects the consumed thread [3,6,20]. Moreover, Jaouachi et al. proved that a heavy fabric consumes more sewing thread than an average fabric. This consumption is estimated for heavy fabrics that are thicker than the average fabrics [20]. According to Sharma et al., fabric thickness has the most important influence in seam consumption because the fabric thickness has a very large range (from 1.64 to $6.56 \mathrm{~mm}$ ) [9]. Nevertheless, Rasheed et al. concluded that the contribution ratio of material thickness was around just 38\% [1].

\subsubsection{Stitch density $\left({ }^{n}\right)$ effect}

Based on Figure 9a-c, stitch density seems an important parameter. In fact, the increase of stitch density from 3 to 5 stitches per $\mathrm{cm}$ increases the thread consumption percentage of $20.41,43.31$, and $31.68 \%$ for 401,402 , and 406 chain stitches, respectively. Thus, the increase of stitch density increases thread consumption. Furthermore, the increase of the consumed amount of sewing thread results from the increase of interlacing zone number inside assembled fabric layers. This result is on agreement with the findings of Rasheed et al. [6] and Jaouachi et al. [20] and confirms our finding that with the fabric feed in motion, the thread is required to form the upper and lower lengths during the stitch length modification. Furthermore, this result is in good agreement with the findings of Lauriol's study [8], which proves that if the sewing length decreases from 2.5 to $2 \mathrm{~mm}$ (4 stitches/cm to 5 stitches $/ \mathrm{cm}$ ), thread consumption increases, approximately by $10 \%$. According to Sharma study, the stitch density presents the second most influential parameter on seam thread consumption, which can be explained by the fact that the stitch density has a small range (from 2 to 3.5 stitches/cm) [9].

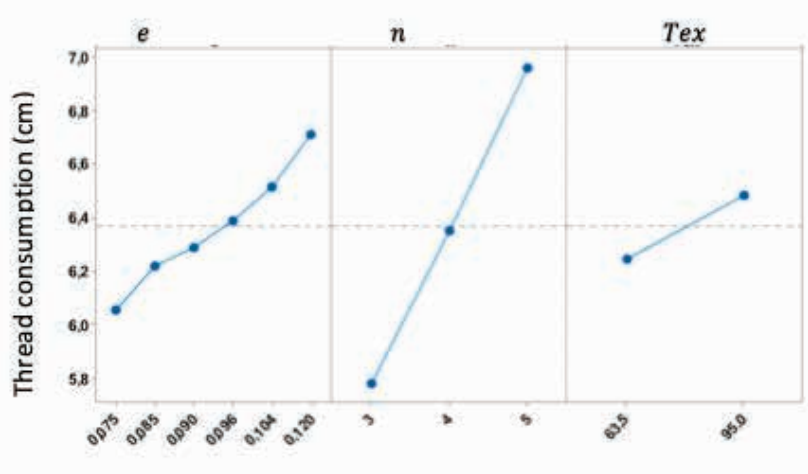

(a)

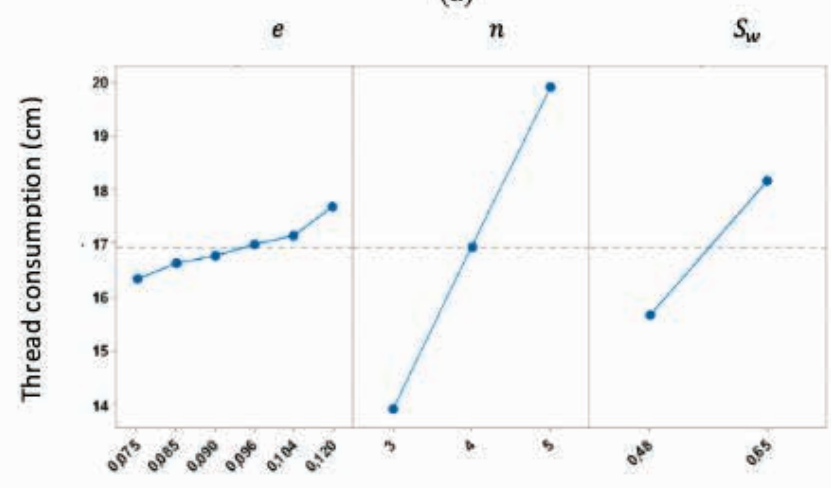

(b)

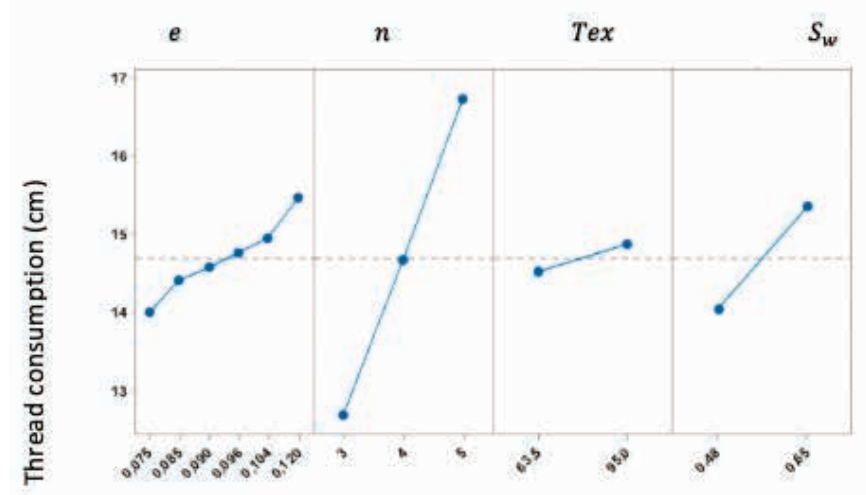

Figure 9. Effects of input parameters for chain stitch type 401 (a), 402 (b), and 406 (c).

Rasheed et al. found that the contribution ratio of stitch density was equal to $62 \%$. In his case, stitch density has an important impact because stitch class 504 is made up of three different threads forming chain shape. Hence, each thread has its own path, but regarding that the path of lopper thread is very curvy, a little increase in stitch density contributes significantly toward the total thread consumption [1].

\subsubsection{Yarn linear density ( Tex ) effect}

Based on Figure 9a-c, we conclude that yarn linear density has not an important impact on thread consumption. In fact, the increase of yarn linear density from 63.5 to 95 tex increases thread consumption by $3.85 \%$ and $2.34 \%$ for 401 and 406 chain stitches, respectively, and it is neglected for 402 chain stitch. This result is on accordance with Jaouachi results [20]. Moreover, if $100 \%$ polyester thread composition is used instead of $100 \%$ cotton yarn, thread consumption becomes less 
significant. Indeed, in the case of polyester thread composition, the increase of the linear density does not have an important effect on thread consumption. Therefore, this result is a good agreement with the properties of each thread.

\subsubsection{Seam width ( $\left.S_{W}\right)$ effect}

According to Figure 8b,c, for 402 and 406, stitch width is an important parameter. The increase of the seam width value $\left(S_{W}\right)$ increases thread consumption. Indeed, increasing $S_{W}$ from 0.48 to 0.65 increased thread consumption by $16.01 \%$ and $9.34 \%$ for 402 and 406 chain stitches, respectively. In our case, the increase percentage value is not very important because the difference between two width levels is not very important, which can explain the low impact of this parameter. This is because the increase of sewing width increases the stitch length. This finding is in a good agreement with the properties reported by Goldnfiber that higher width of seam requires more thread to sew the garments [21].

\section{Conclusions}

The prediction of the required amount of sewing thread using denim samples and chain stitch class 400 using four input parameters through two methods: mathematical (geometrical technique) and statistical (multilinear regressive technique) methods are conducted in this work. Comparing the results, we conclude the accuracy of the geometrical modeling method to explain the consumption behavior in each chain stitch of shape for class 400 .

Indeed, using statistical analysis, multilinear regression models were investigated. Their corresponding $R^{2}$ values ranged from 97.30 to $98.51 \%$, showing their precision. However, using geometrical modeling technique, the $R^{2}$ values ( $R^{2}$ ranged from 98.16 to $99.19 \%$ ) seem more significant than those given by the statistical method. This result encourages industrialists to apply the geometrical models for thread consumption prediction based on the chain stitch of geometry class 400 .

Furthermore, a classification of the significance using Minitab Software is applied to select the input parameters that are most influential on the consumption behavior. In fact, the increase of each parameter increases the seam thread consumption. The parameters that are most influential on the increase of thread consumption are the stitch density followed by stitch width and fabric thickness. The yarn density has the lowest impact on thread consumption values. Stitch density has more impact because stitches of class 400 are made using one, two, or three different threads. Considering that each thread has its own path and the path of lopper thread is very curvy, therefore, a little increase in stitch density contributes significantly toward the total thread consumption.

To conclude, the proposed models, in this study, can accurately predict the thread consumption based on the chain stitch of class 400 due to developed geometrical relationships.

\section{References}

[1] Rasheed, A., Ahmad, S., Ali, N., Rehman, A., Ramzan, M.B. (2018). Geometrical model to calculate the consumption of sewing thread for 504 over-edge stitch. The Journal of the Textile Institute, 2018; doi:10.1080/00405000.2018.14 23902.

[2] Doustar, K., Najar, S. S., Maroufi, M. (2010). The effect of fabric design and weft density on bagging behavior of cotton woven fabrics. The Journal of the Textile Institute, 101, 135-142.

[3] Jaouachi, B., Aouine, S., Khedher, F. (2017). Consumed sewing thread behaviour based on Lockstitch and chain

stitch. Indian Journal of Fibre \& Textile Research, 42, 325-334.

[4] Khedher, F., Jaouachi, B. (2014). Waste factor evaluation using theoretical and experimental jean pants consumptions. The Journal of the Textile Institute, 106, 402-408.

[5] Jana, P. (2011). Assembling technologies for functional garments-An overview. Indian Journal of Fibre \& Textile Research, 36, 380-387.

[6] Rasheed, A., Ahmad, S., Mohsin, M., Ahmad, F., Ali Afzal, M. (2014). Geometrical model to calculate the consumption of sewing thread for 301 Lockstitch. Journal of Fibre \& Textile Research, 105, 1259-1264.

[7] Webster, J., Laing, R. M., Niven, B. E. (1998). Effects of repeated extension and recovery on selected physical properties of ISO-301 stitched seams, part I: load at maximum extension and at break. Textile Research Journal, 68(11), 854-864.

[8] Lauriol, A. (1999). Modes and techniques: Initiation to the technology of materials in the clothing industries. 2nd ed. France: Strasbourg.

[9] Sharma, S., Gupta, V., Midha, V. K. (2017). Predicting sewing thread consumption for chain stitch using regression model. Journal of Textile Science and Engineering, 7, ISSN 2165-8064.

[10] Vinay, K. M., Shailja, S., Vaibhav, G. (2016). Predicting sewing thread consumption for lockstitch using regression model. Research Journal of Textile and Apparel, 20, 155163.

[11] Jaouachi, B., Khedher, F. (2015). Evaluation of sewed thread consumption of jean trousers using neural network and regression methods. Fibres \& Textiles in Eastern Europe, 111, 91-96.

[12] Jaouachi, B., Khedher, F. (2013). Evaluating sewing thread consumption of jean pants using fuzzy and regression methods. The Journal of the Textile Institute, 104, 10651070.

[13] Ghosh, S., Chavhan, Md. V. (2014). A geometrical model of stitch length for lockstitch seam. Indian Journal of Fibre and Textile Research, 39(2), 153-156.

[14] Jaouadi, M., Msahli, S., Babay, A., Zitouni, B. (2006). Analysis of the modeling methodologies for predicting the sewing thread consumption. International Journal of Clothing Science and Technology, 18, 7-18.

[15] Midha, V. K., Sharma, S., Gupta, V. (2016). Predicting sewing thread consumption for lockstitch using regression model. Research Journal of Textile and Apparel, 20(3), 155-163. 
[16] Gazzeh, M., Khedher, F., Jaouachi, B. (2017). Modelling the sewing thread consumption of 602 cover-stitch based on its geometrical shape. International Journal of Applied Research on Textile, 5, 1-15.

[17] NFG 07-117. (1981). Method of assessing of fabric threads slippage and measurement of the seam resistance. French Association for Standardization AFNOR. 201-209.

[18] Md Sohanur Rahman Sobuj. (2015). Relation between yarn count and diameter \& count calculation for ply yarn. Web site: https://textilestudycenter.com/relation-betweenyarn-count-and-diameter/ (27 February 2015).
[19] Gazzeh, M. (2015). Study of the effect of wire/wire friction on the behavior of Denim tissue poaching. PhD thesis in Textile Engineering, University of Monastir. Tunisia.

[20] Jaouachi, B., Khedher, F., Mili, F. (2012). Consumption of the sewing thread of jean pant using Taguchi design analysis. Autex Research Journal, 12, 81-86.

[21] Goldnfiber. (2018). Major factors for doing sewing thread consumption. Web site: http://www.goldnfiber. com/2016/07/major-factors-for-doing-sewing-threadconsumption.html. 